\title{
Postgraduate Teaching Research under the Platform of CEEUSRO Sheliang Wang ${ }^{a}$ and Shimin Zhang ${ }^{\text {b, }}$

\author{
School of Civil Engineering, Xijing University, Xi'an 710123, China
}

\author{
Keywords: CEEUSRO; Intermediary organization; Joint training model; Market demand
}

\begin{abstract}
The science、 technology and society are becoming more and more integrated at present and the rapid development of knowledge economy has put forward higher requirements for innovation, so innovation has become the main driving force for the development of science and technology and society. The development of the world today can't rely solely on the efforts of one side, but the interaction and cooperation. This paper analyzes in the construction of intermediary organization、 joint training mode and market demand analysis of the three factors of the development through the research of domestic and international cooperation in this background to enhance the capability of independent innovation 、 enhance international competitiveness 、 promote the interaction between science and technology and economic development, providing theoretical and practical significance of construction of national innovation system.
\end{abstract}

\section{Introduction}

The joint training of postgraduate education is an important measure for colleges and universities to accumulate educational resources and expand educational space and break through the bottleneck of education. It is an inevitable choice for the training of innovative talents in the era of knowledge economy. Also it is an effective way to realize the coordinated development of graduate education scale and structure with the economic and social development. However, the development of the joint training of postgraduate education is short, the mode and mechanism are immature, and many problems have been exposed in the process of cognition and practice, which seriously restricted their healthy development[1-2].

\section{Development and Present Situation of Graduate School Teaching}

Since the middle of the twentieth Century, with the rapid development of science, technology economy and society, a large number of cross disciplines have emerged in the new scientific and technological revolution, which have made rapid progress in that. In order to integrate and make full use of scientific and technological resources and give full play to the role of science and technology in promoting the economy and society, the concept of CEEUSRO has first appeared in developed countries in the world[3].

Kazumasa Kawasaki mainly investigated the cooperation of Japan's small and medium-sized enterprises through some cases to summarize the successful co-operation education strategies, he uses practical methods in the specific research to study and analysis cooperation mod between University researchers and enterprise. Koschat had purposed the concept that transfer of technology in production research cooperation is essentially a knowledge transfer[4-5]. Etzkowitz and Leydesdorff. had purposed the three spiral theory paradigm which has aroused widespread concern of Chinese scholars abroad. Three screw theory puts knowledge as innovation, and government、 industry and university integrate as a cohesive force to promote knowledge into productivity, at the same time, all of that still retain their own characteristics and do not lose their independence. At present, the focus of domestic scholars is how to overcome the "acclimatized" of this foreign theory, combining with China's reality through two aspects of theory and practice.

It is the internal requirement of talent growth to carry out joint production、 research and production of graduate students. Zhang Lian (1994) pointed out that "cooperative education for applied talents cultivation in Colleges and universities is actively adapt to the social needs of the 
effective way", based on the situation of the shortage of teachers and researches from analyzing Chinese enterprises on the application demand of high level talents and cultivating applied talents . Zhong Binglin (2000) insisted that promoting the cooperative education of CEEUSRO is the need of universities and enterprises to develop themselves and an effective way to promote quality education.

Wu Shiming and Shi Zhenglan (2000) analyzed complementary university system and the enterprise system from the perspective of the cultivation of innovative talents. They researched on the macro and specific operational level and pointed out that "cooperative education is an inevitable choice for cultivating innovative talents". Ye Xiaoyan (2000) pointed out that the training mode of research without CEEUSRO will cause students"go out of school and be at a loss what to do", "get nothing of knowledge"and "lower overall quality", also at same time the development of education is not to support the industry which unfavorable to the development of education[6-7]. In order to overcome these shortcomings together with the train high-quality engineering and technical talents, it is important to combine classroom teaching with engineering research training and follow the way of cooperative education.

In the book of "the graduate education mode of", Li Shengbing proposed that "collaborative" graduate training mode is the past of postgraduate training mode "breakthrough and change" and is the product of new technological revolution and the social and economic development after the second world war. It is plays a very important role in the applied research and training of talents and in the teaching and scientific research, production combination, which promotes the university and the society of actual production and life is more extensive and in-depth contact. He also said that combination of scientific research and production is the key link to train applied research talents, combining with teaching and production and embodying the essential characteristics of collaborative graduate education model.

Xiao Guofang (2007) also pointed out that "the study of the combination of the training mode is that enterprises , universities research institutes and other production education and scientific research subject in order to achieve high level personnel training objectives and specifications and quality, integrating production 、 teaching and scientific research into the knowledge ability and quality structure for graduate students and realizing the overall operation of this structure according to the law and the social high level talents 、 production 、 teaching and research integration[8].

Above of cooperative graduate education discussion on the essence of showing the deepening of the characteristics from the combination of teaching scientific research and production to combination of outstanding educational center, it reflects on the understanding of the deepening and ensure the starting point of cooperative education of graduate students and the destination. It lays a foundation for further research on the relationship among the three functions of teaching and research、 production and research and guiding the practice reform.

\section{Promote Graduate Students' Joint Training Strategy}

Strengthen the Intermediary Organization Construction of CEEUSRO to Graduate Students. The most important invention of higher education in the twentieth Century was the organizational form, which moderated the main structure of centralized control by means of intermediary organizations. Graduate cooperative education also need such intermediary organizations to coordinate between universities industry and scientific research institutes of the different needs and interests, in order to resolve conflicts every aspects of CEEUSRO and provide professional consulting services.

According to the characteristics of situation that CEEUSRO together with joint training is at the stage of active exploration of postgraduate education in China, the government could establish a joint training committee which is responsible for joint training for postgraduate education and research. Postgraduate joint training steering committee of CEEUSRO can be consisted by universities 、 research institutes and industry representatives, and the pattern of England's 
implementation of the "coordinator" system also can be taken, like assigning coordinators to colleges and universities industry and research institutes, and guiding the practice of joint production、 research and production of graduate students in every subjects.

The main function of the postgraduate joint training steering committee of CEEUSRO is what shows down:

Organization and Implementation. The committee takes over the role of the organizer of the government and tries to develop channels for research v production、 research and joint training, building training bases and providing support for joint training practices also.

Communication and Coordination. The committee should set up a bridge for effective cooperation and communication between production、study and research, and information can be effectively transmitted. So that the contradictions in the process of production and research can be effectively solved to ensure the smooth progress through that.

Monitoring and Identification. The committee should always focus on educating people and strengthen the process supervision of research 、 production、 research 、 research and joint training to discover and solve problems at once. It is necessary to formulate an assessment index system for research、 production、 research and training, evaluating and identifying the projects 、 bases and achievements and ensuring the efficiency and effectiveness of educating people.

Construct and Innovate Joint Training Model of based on Development of Students. The key to the effectiveness of postgraduate education is to build a joint education model of educating people. At present, the "people oriented" concept is difficult to effectively implement in practice, resulting in joint training model of CEEUSRO can't consciously developed towards direction of the centre of scientific research and economic interests. So it needs to innovate and construct the joint training mode suitable for the talent training based on the characteristic of the university itself.

First of all, training mode needed to be selected according to the training target positioning. Due to the characteristics of the development of high-tech industry, a breakthrough in basic research is becoming more and more popular with the industry direct attention, making the cooperative graduate education is not limited to the cultivation of applied talents, but to the diversification of talents training target transformation. Specifically, the research of academic talents joint training is suitable for the introduction of strategy, making social resources into the existing postgraduate training system that centered in laboratory and research. The training of applied and technical talents is more suitable for the social and industry oriented joint training model and the actions of "go outside" needed to be done. In addition, we should respect the development status of students, and give them the power of independent choice and training according to the students' characteristics and development trends. At the same time, it is necessary to build a channel of mutual transformation among various training modes, and strengthen the opening and compatibility of the model. If students can't adapt to a certain training mode, they can continue to learn in other modes.

Secondly, we should further enhance the openness of the curriculum system and pay attention to the interaction with the development of the industry. The study of production 、 research and joint training mode should be further strengthened in industry front knowledge on the basis of the existing elective system and further in specific teaching content and teaching methods. The latest theoretical research results of industrial development will be introduced into the curriculum system in real time. We should pay attention to the training of graduate students to meet the needs of cross disciplinary studies, and reform the curriculum system with the principles of applying what we have learned and exerting the initiative and exploring spirit of students.

Finally, we should reform the evaluation system and pay attention to creating a comprehensive environment for educating people. The evaluation system plays a guiding role and also has the function of finding problems and correcting errors. The evaluation of students should not only focus on the research results, but also make a comprehensive evaluation of the students' comprehensive literacy, so as to reflect the possible drawbacks of the current model. At the same time, we need to realize concept that good education environment is good to students, integrating school 
environment with social environment well in the process of construction of cooperative graduate training model. On the one hand, students' development issues under the single school environment need to be overcome ; on the other hand, we need to control the negative effects that social environment may caused in process of the growth of students, strengthening the control of the process to shape students' healthy character and all-round develop their social psychology and social role[9].

Strive to Promote Integration of Scientific Research with joint Training Model of CEEUSRO based on Market Demand Closely. Educational foresight is achieved only by understanding industry needs. Whether we can keep up with the market demand and carry out joint training of postgraduate education is the key point for graduate students to adapt to the future competition in science and technology. The repetitive development from the market front is unable to cultivate talents with innovative spirit and creative consciousness. This requires colleges and universities should have the forefront of consciousness, the consciousness of education and abandon some low-end development project economic temptation to make maximum benefits of joint training when conducting joint training in scientific research projects.

Where is the market,there exists motivation and this is a long-term mechanism to solve the problems of joint training model of CEEUSRO to postgraduate. Market demand determines the direction of scientific research development, and also determines the social and economic value of scientific research achievements. Higher education research results can only be known to few when they do not connect with each other, also cannot create value for economic and social development, ay same time, also can't receive strong support from outside in this way. It is also true that the joint training of CEEUSRO to postgraduate is closely related to market demand and is a prerequisite for industrial support.

The integration of scientific research and joint training of CEEUSRO to postgraduate is an important measure to make the research resources and graduate education resources get a macro and effective allocation. The university should not only in the implementation of scientific research and postgraduate co culture, but also make corresponding responses like professional setting v the scale and structure of joint training according to the development direction of the whole society. In the specific process of joint training, according to the carrying capacity of scientific research projects, the scale of joint training of production, research and research can be studied to ensure the quality of joint training and improve the efficiency of joint training[10].

\section{Conclusions}

In the background of the current social science and technology integration and knowledge economy, it is important to improve the ability of independent innovation and enhance international competitiveness and achieve sustainable development through cooperation to integrate resources in the post crisis era in order to respond the construction of an innovative country .

The cooperative joint training model provides useful reference for our research in the aspects of intermediary service、market demand and system construction;

The three-in-one relationship of joint training of CEEUSRO makes the educational status vulnerable to scientific research and service function, which need to transform concept into specific patterns through the construction of organization and system ,to make the joint training of CEEUSRO to postgraduate institutionalized due to demands of the talents and turn interest driven into quality driven.

\section{Reference}

[1] Wang Li. Graduate Education Innovation Center: a new model of industry university cooperation, [J]. intelligence, 2011, (01): 28-29.

[2] Zhang Wendong. Government, industry, research, industry and trade linkage. Promote the construction of graduate education innovation center. [J]. degree and graduate education, 2011, (01): 20-24. 
[3] Tu Ya Qing, Mao Yuwen, Yang Huiyue. The cooperative education of government, industry, research, production and training. Innovative ability of graduate students [J]. China Electric Power Education, 2011, (26): 56-58+62.

[4] Liu Junyue, Huang Wei Yang Xiaorui, nine, more than Yunsheng. Train coupling research mode $[\mathrm{J}]$. Journal of Chongqing Institute of Technology graduate education and Research (SOCIAL SCIENCE EDITION), 2009, (02): 155-157.

[5] $\mathrm{Hu} \mathrm{Yu} \mathrm{Yu.} \mathrm{Combination} \mathrm{of} \mathrm{production,} \mathrm{research} \mathrm{and} \mathrm{research,} \mathrm{and} \mathrm{promote} \mathrm{the} \mathrm{level} \mathrm{of} \mathrm{graduate}$ education in futures major [J]. Journal of Zhejiang Gongshang University, 2010, (02): 88-90+96.

[6] Liu Jia. Research on joint training mechanism of postgraduate education in local universities [D]. Guangxi Teachers Education University, 2010.

[7] Wei Wei. The training mode of graduate students in local universities. Exploration of cooperative education of production, research and research [J]. Journal of the National Institute of education administration, 2008, (09): 65-68.

[8] Liu Siyuan, Xie Qing. The Nissan research cooperation mechanism of Guangxi local colleges and universities graduate education implications [J]. Science (a), Wenhui 2014 (01): 3-5.

[9] Li Li, Kong Jianqiong. Taking the postgraduate training as an opportunity to promote the development of graduate education in the hospital. [J]. Chinese medicine Herald, 2014, (14): 130-133.

[10]Liao Xiaomei, Wei Feng. Construction of training model of collaborative innovation in graduate education [J]. education and profession, 2016, (18): 31-33. 